\title{
GeneData Screener
}

National Cancer Institute

\section{Source}

National Cancer Institute. GeneData Screener. NCI Thesaurus. Code C123889.

Proprietary software that is designed to import data from any screening instrument and analyze and manage all types of screening data. 